\title{
Les débuts de la Société des Missions-Étrangères de la Province de Québec (1919-1931)
}

\section{Jules Bernard}

Volume 38, 1971

La genèse de la Société des Missions-Étrangères de la province de Québec

URI : https://id.erudit.org/iderudit/1007264ar

DOI : https://doi.org/10.7202/1007264ar

Aller au sommaire du numéro

Éditeur(s)

Les Éditions Historia Ecclesiæ Catholicæ Canadensis Inc.

ISSN

0318-6172 (imprimé)

1927-7067 (numérique)

Découvrir la revue

Citer cet article

Bernard, J. (1971). Les débuts de la Société des Missions-Étrangères de la Province de Québec (1919-1931). Sessions d'étude - Société canadienne d'histoire de l'Église catholique, 38, 31-54. https://doi.org/10.7202/1007264ar

Tous droits réservés @ Les Éditions Historia Ecclesiæ Catholicæ Canadensis Inc., 1972
Ce document est protégé par la loi sur le droit d'auteur. L'utilisation des services d’Érudit (y compris la reproduction) est assujettie à sa politique d'utilisation que vous pouvez consulter en ligne.

https://apropos.erudit.org/fr/usagers/politique-dutilisation/ 


\section{Les débuts de la Société des Missions-Etrangères de la Province de Québec (1919-1931)*}

En la fête de la Purification, le 2 février 1921, le cardinal Bégin présidait à l'archevêché de Québec une réunion des évêques de la province de Québec. $M^{\mathrm{gr}}$ Paul-Eugène Roy, coadjuteur du cardinal, $\mathbf{M}^{\mathrm{gr}}$ Paul Laroque, évêque de Sherbrooke, $\mathbf{M}^{\mathrm{gr}}$ Brunault, évêque de Nicolet, $M^{g r}$ Bernard, évêque de Saint-Hyacinthe, $M^{g r}$ Latulipe, évêque de Haileybury, $M^{g r}$ Brunet, évêque de Mont-Laurier, $M^{g r}$ Léonard, évêque de Rimouski, $\mathbf{M}^{\mathrm{gr}}$ Gauthier, auxiliaire de Montréal et $\mathrm{M}^{\mathrm{gr}}$ Forbes, évêque de Joliette, assistaient à cette réunion. Au cours de cette assemblée, le cardinal Bégin exposa le désir du pape et de la Sacrée Congrégation de la Propagande.

de voir le Canada français prendre avec les autres nations catholiques, dans le champ de l'apostolat auprès des infidèles, une place en quelque sorte officielle non seulement en envoyant de nombreux sujets, hommes et femmes, dans les diverses sociétés de missionnaires, ce qu'il fait déjà noblement, mais en ayant sous la tutelle de ses évêques et sous la direction de la Propagande, un Séminaire canadien des MissionsEtrangères.

Les évêques décidèrent ensuite à l'unanimité de fonder un séminaire canadien des Missions étrangères dans la province de Québec et, pour donner suite à cette décision, ils élirent un Comité qui serait responsable de l'établissement de cette oeuvre. Ce Comité se composait de $\mathrm{M}^{\mathrm{gr}}$ Paul-Eugène Roy, coadjuteur à Québec, $\mathrm{M}^{\mathrm{gr}}$ Brunet, évêque de Mont-Laurier, et $\mathrm{M}^{\mathrm{gr}}$ Forbes, évêque de Joliette ${ }^{1}$.

La décision de l'épiscopat d'établir un Séminaire des Missions Étrangères serait communiquée officiellement à toute la province de Québec par la publication d'une lettre collective des évêques le 23 avril 1922. Cette lettre fut lue dans toutes les églises, le dimanche de Quasimodo.

Dès le 3 février, le Comité d'organisation du séminaire se réunit à l'archevêché de Québec. $M^{\text {gr }}$ Roy fut choisi comme président du Comité et $M^{8 r}$ Forbes comme secrétaire. $M^{8 r}$ Gauthier remplaça $M^{8 r}$ Bruchési. Le Comité prit les premières décisions qui s'imposaient pour la fondation de cette œuvre. Il décida de fonder une Société qui serait reconnue par le gouvernement de la province de Québec sous le nom de Corporation du

* C'est grâce à M. l'abbé Lucien Lemieux, de la faculté de Théologie de L'Université de Montréal, que nous pouvons profiter de la thèse toute récente (1970) du Père Jules Bernard, qui a lui aussi autorisé la publication des extraits qu'on lira ici.

1 ASMEQ, DPVC. 
Séminaire Saint-François, ce saint étant le patron des missions et le patron secondaire de la province. Le séminaire serait à la charge des évêques de la province. Montréal serait l'endroit idéal pour l'établissement de celui-ci, d'abord en raison des facilités de communication avec les grands centres et aussi pour les avantages dont la Société pourrait bénéficier en étant en relation plus étroite avec les Sœurs de l'Immaculée-Conception qui œuvraient en Chine, et qui s'occupaient des Chinois établis à Montréal, car c'était vers la Chine que les évêques désiraient voir aller leurs missionnaires ${ }^{2}$. Le Comité demanda aux évêques de voir si, dans leurs diocèses, il y avait des prêtres qui accepteraient de se dévouer à cette œuvre. Quelques prêtres s'étaient déjà offerts et le Comité vit à les employer à la préparation de l'établissement matériel de l'œuvre. Comme la Propagation de la Foi allait devenir la principale source de revenu du séminaire, il fallait réorganiser celle-ci. Un procès-verbal de chacune des réunions du Comité d'organisation serait envoyé aux évêques pour qu'ils suivent l'évolution de l'œuvre ${ }^{3}$.

Le 24 février 1921, le secrétaire du Comité, $\mathrm{M}^{\mathrm{gr}}$ Forbes, communiqua au cardinal Van Rossum, préfet de la Propagande, la décision des évêques d'établir un Séminaire des Missions Étrangères et les décisions subséquentes du Comité d'organisation. Il demanda l'autorisation de procéder à cette fondation et les directives de la Propagande à ce sujet ${ }^{4}$. Le cardinal Van Rossum félicita les évêques de leur initiative qui arrivait en temps opportun, et il donna l'autorisation demandée ${ }^{5}$.

A l'occasion des séances du Conseil de l'Instruction publique, le 11 mai 1921, les évêques qui se trouvaient à Québec se réunirent à l'archevêché et décidèrent que le moment était venu de nommer le supérieur du séminaire. Le chanoine Ovila Roch, curé de la cathédrale de Joliette, fut choisi pour occuper ce poste. Ils donnèrent aussi les noms de $M^{\mathrm{gr}}$ Ross, vicaire général de Rimouski, du chanoine Gignac de Québec et de l'abbé Lapierre de Montréal, comme collaborateurs éventuels ${ }^{6}$.

\section{1 - Établissement et organisation financière de la Société}

L'important était d'abord de regrouper les principaux collaborateurs afin qu'ils puissent avantageusement coordonner leurs efforts dans la réalisation de ce projet. Pour ce faire, il fallait faire l'acquisition d'un logement le plus tôt possible. Dès le mois de mai 1921, le chanoine Roch se mit à la recherche d'un immeuble qui pourrait servir comme séminaire. Deux immeubles avaient retenu l'attention, mais aucune transaction ne fut conclue, vu leurs prix élevés. Le supérieur demeura à Joliette

\footnotetext{
Ibid. Procès-verbal de la première réunion.

Ibid., 17 février 1921, deuxième réunion.

ASMEQ, $A M E$, 11-14, Forbes à Van Rossum (copie).

Ibid., 15-17, 13 avril 1921, Van Rossum à Forbes (copie)

ASMEQ, DPVC, 12 mai 1921, troisième réunion.
} 
jusqu'au mois d'août pour venir ensuite s'installer à Montréal, dans la maison provinciale des Clercs Saint-Viateur qui avaient mis une petite chambre à sa disposition. Les autres collaborateurs attendirent dans leurs diocèses jusqu'à ce que la nouvelle œuvre trouve un refuge ${ }^{7}$. Cet heureux événement se produisit au mois de septembre 1921. En effet, la direction provinciale des Clercs Saint-Viateur mit à la disposition des évêques, pour le séminaire, une de leurs propriétés, l'ancien presbytère d'Outremont. Avec quelques améliorations, cette maison pourrait facilement abriter les directeurs et une dizaine de séminaristes. Elle fut prêtée sans autres frais que de voir à son entretien et de payer les impôts qui l'affectaient. Quelques jours après, le chanoine Roch, ainsi que les abbés Rondeau et Lapierre, vinrent prendre possession de leur nouvelle demeure ${ }^{8}$.

Entre temps, le cornité d'organisation pour le séminaire avait décidé que les évêques, pour venir en aide à la nouvelle fondation, verseraient " chaque année une somme proportionnelle au nombre de leurs diocésains, soit un sou par catholique ». Cette contribution allait fournir au séminaire une somme d'environ vingt mille dollars par année. Un premier versement devait être fait avant la fin de l'année courante. Les évêques pouvaient s'acquitter de leur obligation, en prélevant sur les fonds de la Propagation de la Foi, ou par une quête pouvant atteindre la somme requise. Cet argent devait être envoyé au secrétaire du Comité ${ }^{\vartheta}$. Les contributions rapportèrent, pour les mois d'octobre, novembre et décembre de l'année 1921, le montant de trois mille cinq cent douze dollars. Pour être en mesure de faire face aux besoins nouveaux qui s'annonçaient, le Comité recommanda de faire le plus tôt possible le versement du premier quart de l'année nouvelle ${ }^{10}$.

En effet, la maison d'Outremont n'étant pas suffisamment grande pour recevoir les aspirants, de nouvelles démarches avaient été entreprises pour trouver un immeuble plus convenable. Trois sites furent soumis à l'étude du Comité d'organisation. Un premier site, la propriété de Bordeaux, appartenant aux Clercs Saint-Viateur, comprenait dix arpents de terre et une maison construite mais à transformer. Elle ne fut pas agréee. Le Comité refusa aussi l'offre du collège de Saint-Hubert dans le comté de Berthier, à soixante milles de Montréal. L'accord se fit sur la propriété Desnoyers qui comprenait vingt arpents sans construction. Elle était située dans la municipalité de Saint-Vincent-de-Paul, aux abords de Pont-Viau et à proximité des tramways de Montréal. Elle offrait comme site et comme étendue des avantages précieux ${ }^{11}$. M. le supérieur entreprit

7 Ibid., 28 septembre 1921, quatrième réunion.

8 Ibid.

9 Ibid.

10 Ibid., $1^{\text {er }}$ février 1922, cinquième réunion.

11 Ibid. 
donc les négociations pour l'acquisition de ce terrain, aux meilleures conditions possibles. En vertu des pouvoirs conférés par la charte civile d'Incorporation de la Société, votée le 6 mars 1922, le Conseil d'administration du séminaire, qui prend la relève du Comité d'organisation, décida, le 26 avril 1922, d'acheter au prix de vingt mille dollars, payables comptant, un terrain de vingt arpents, propriété de la Succession Mathias Desnoyers. Un rabais de cinq mille dollars avait été consenti par la succession. Le contrat fut signé le lendemain devant maître A. Gervais, notaire commissaire de Montréal ${ }^{12}$. Quelques mois après, la Société fit l'acquisition d'un terrain voisin à celui déjà acheté, au prix de huit mille dollars. Il était la propriété de M. Joseph Chaput ${ }^{13}$.

Les achats de terrains avaient diminué le compte en banque, et il fallait de nouvelles sources de revenus pour la construction du séminaire, qui devait commencer en juillet. Le chanoine Roch, avec l'autorisation du Conseil d'administration, emprunta à la Fabrique de la paroisse Saint-Elisabeth une somme de cinq mille dollars, par billet à un an, avec intérêt de cinq pour cent. Un billet de sept mille dollars, à cinq pour cent, payable à l'Ordre des Sours Adoratrices du Précieux-Sang, fut transféré à la Société des Missions Étrangères. Un autre prêt de trois mille dollars, à fonds perdu, fut consenti par le curé de la paroisse de Saint-Martin ${ }^{14}$. Ces emprunts et quelques autres sources de revenus permirent d'entreprendre, en juillet 1922, la construction des fondations et du sous-sol du séminaire ${ }^{15}$. En septembre, les dépenses de la construction s'élevaient à neuf mille dollars. Il restait en caisse vingt-huit mille dollars. Le Conseil d'administration jugea à propos de faire poursuivre les travaux de construction. La Société fit de nouveaux emprunts, au total, trente sept mille dollars ${ }^{16}$.

Le 16 octobre 1922, le délégué apostolique au Canada et à TerreNeuve, $M^{\mathrm{gr}}$ Pietro di Maria, présida la cérémonie de la bénédiction solennelle de la pierre angulaire du Séminaire Saint-François-Xavier, en présence d'un grand nombre d'évêques de la province de Québec et des autres provinces, qui se trouvaient alors à Montréal pour les fêtes jubilaires de la Saint-Patrick. Le cardinal Bégin de Québec et $\mathbf{M}^{\mathbf{g r}}$ Bruchési de Montréal, ainsi que leurs évêques coadjuteurs et les évêques de Chicoutimi, Sherbrooke, Trois-Rivières, Nicolet, Saint-Hyacinthe, Haileybury, Pembroke, Joliette, Rimouski, Mont-Laurier et plusieurs évêques du Canada et des États-Unis assistèrent à la cérémonie ${ }^{17}$. Le clergé et les fidèles répondirent aussi en grand nombre à l'invitation qui leur avait été faite

12 Ibid., 26 avril 1922, sixième réunion.

13 Ibid., 11 juillet 1922, huitième réunion.

14 Ibid.

15 Ibid.

16 ASMEQ, AME, 53-55, 27 septembre 1922.

17 Ibid., 55-59. 
par la voix des journaux et de la Semaine Religieuse de Québec. Environ quatre cents prêtres et religieux, et quelques milliers de fidèles, y participèrent ${ }^{18}$.

Dans le discours qu'il prononça à cette occasion $\mathbf{M}^{\mathrm{gr}}$ Roy, coadjuteur à Montréal, rappela que cette ouvre était en lien direct avec la mission que le Christ avait donnée à ses apôtres d'aller porter la bonne nouvelle de salut à toutes les nations. L'Église canadienne voulait assumer sa responsabilité de porter aux peuples païens la foi qu'elle avait si généreusement reçue, car \& la foi qui n'est pas apostolique est une foi stérile ». De plus, le Canada connaissait des heures difficiles, car certains mauvais courants de pensée avaient fait baisser la foi et les mœurs dans plusieurs milieux. Or, disait $\mathbf{M}^{\mathrm{gr}}$ Roy,

le meilleur moyen de garder la foi et les mours, c'est de porter la foi ailleurs. Il existe une étroite relation entre le don de la foi reçue et de la foi communiquée. Voilà pourquoi l'Eglise catholique québécoise entre dans cet esprit apostolique.

$\mathrm{M}^{\mathrm{gr}}$ Roy invita la population à venir en aide à cette cuvre officielle de l'Église du Canada français en favorisant les vocations missionnaires. Dans les collèges et les couvents, de nombreux jeunes désiraient se vouer à l'apostolat. "Que les fidèles se gardent bien de mettre obstacle à cette ardeur. Le prêtre n'est jamais plus dans l'esprit du sacerdoce que sur le chemin de l'apostolat ", disait-il. La population pouvait aussi aider l'œuvre en fournissant les revenus suffisants pour son développement ${ }^{19}$. Cet événement célébré avec tant de solennité était très significatif de l'importance que revêtait cette œuvre aux yeux de la hiérarchie et des fidèles de la province. D'ailleurs, le discours de $\mathbf{M}^{\text {gr }}$ Roy montrait bien jusqu'à quel point il était dans l'intérêt de l'Église canadienne de soutenir cette cuvre.

Pendant que la construction du séminaire se poursuivait, certaines organisations furent mises sur pied pour aider la Société. En mars 1923, un groupe de dames décida de former une union pour le soutien du séminaire sous le patronage des saintes femmes pourvoyeuses du collège apostolique. Cette initiative était due à la supérieure générale des Sœurs de l'Immaculée-Conception.

Quelque temps après, la Société organisa son propre système d'aide au séminaire en accordant des titres et avantages à ses bienfaiteurs. A la fin de l'année 1923, soit deux ans et quelques mois après la fondation du séminaire, les dons et quêtes s'élevaient à la somme de trente-six mille dollars et les prêts à fonds perdus à la somme de cinquante et un mille dollars. La contribution des diocèses avait rapporté quarante-trois mille trois cents dollars, soit en tout cent trente mille dollars ${ }^{20}$.

18 Ibid., 54.

19 ASMEQ,AJS, 17 octobre 1922, Le Devoir.

20 ASMEQ, SSME, 75. 
Les travaux du séminaire se terminèrent le 21 février 1924. La construction avait coûté trente mille dollars, sans compter l'achat du terrain. M. Monette, architecte et M. Phaneuf, contremaître, en avaient dirigé les travaux. Le 27 février, le supérieur et ses collaborateurs vinrent prendre possession de leur résidence ${ }^{21}$. La bénédiction du séminaire eut lieu le 7 septembre 1924, veille de la Nativité de la Vierge, en présence d'une dizaine d'évêques de la province de Québec, des représentants des gouvernements provinciaux et fédéraux, des autorités municipales ainsi que d'un grand nombre de prélats, de supérieurs de séminaires, collèges et instituts religieux et de plusieurs prêtres et fidèles. $M^{\text {gr }}$ di Maria, délégué apostolique, présida la cérémonie ${ }^{22}$.

Pour assurer les ouvrages intérieurs et manuels du séminaire, des démarches avaient été faites auprès des Sœurs de Saint-Antoine-de-Padoue de Chicoutimi. Elles acceptèrent avec plaisir de collaborer à cette œuvre. Dès le mois de septembre, elles étaient au poste, au nombre de cinq. Une somme de cent dollars par année était exigée pour chaque sour ainsi que les frais de voyage ${ }^{23}$.

Au point de vue financier, la Société était maintenant assurée de trouver les revenus nécessaires à son développement. L'œuvre avait aussi un logement pour abriter son personnel. Dès le mois de septembre, les aspirants vinrent habiter au séminaire où ils reçurent la formation théologique nécessaire à leur apostolat missionnaire. Sur la plan matériel, l'œuvre était lancée et elle allait pouvoir se développer normalement suivant les exigences du progrès.

\section{2 - Recrutement et formation des aspirants}

Tout en travaillant à l'organisation matérielle du séminaire, le chanoine Roch et ses collaborateurs ne négligèrent pas le recrutement des vocations. Les premiers aspirants se présentèrent à peine quelques mois après la décision de l'épiscopat d'établir le Séminaire des Missions Étrangères. Le 30 mai 1921, Léo Lomme, élève au Séminaire de Philosophie de Montréal, fit sa demande d'admission au chanoine Roch. Un autre élève du Séminaire de Philosophie demanda son admission au chanoine Roch le 12 juin suivant ${ }^{24}$. Cependant, comme la Société ne possédait point de maison pour donner le cours de théologie, $\mathrm{M}^{\mathrm{gr}}$ Forbes demanda au supérieur du Grand-Séminaire de Montréal de bien vouloir admettre les deux aspirants en première année de théologie $^{25}$. Le supérieur du Grand-Séminaire accepta avec plaisir de rendre ce service à la nouvelle Société ${ }^{26}$.

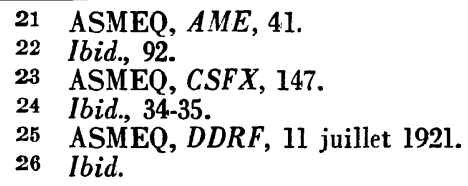


Mais les vocations n'allaient pas toutes venir d'elles-mêmes, il faudrait aller les chercher. Aussi le 17 janvier 1922, le chanoine Roch partit pour faire la visite des collèges et séminaires de la province, parler des missions à la jeunesse étudiante et montrer les avantages que la Société offrait aux Canadiens français qui n'auraient plus à frapper à la porte des communautés étrangères à leur nationalité, et à leurs aspirations pour aller travailler dans les pays de missions.

En plus des tournées de propagande dans les collèges, des conférences furent publiées sous forme de tracts. La première s'intitulait: Vers les terres d'infidélité et la seconde: Autour du Séminaire canadien des Missions Étrangères. Ces tracts furent envoyés par milliers d'exemplaires aux étudiants et au clergé de toute la province. Plusieurs autres tracts parurent les années suivantes ${ }^{27}$.

La propagande se fit aussi par l'intermédiaire des journaux qui publièrent de nombreux articles sur le développement de l'œuvre et sur les missions ${ }^{28}$. Certains événements, comme le trois centième anniversaire de la Propagande et le centième anniversaire de la Propagation de la foi, donnèrent l'occasion d'intéresser la population à l'œuvre missionnaire. Pie XI avait demandé pour cette circonstance qu'on fasse dans le monde entier des prières et des prédications en faveur des missions. Les évêques canadiens entrèrent dans le mouvement et commandèrent dans leurs diocèses des triduums de prières, de pénitence et de prédications en faveur des missions. Les prêtres des Missions Étrangères prêchèrent ces triduums dans une douzaine de paroisses ${ }^{29}$.

Deux ans après sa fondation, le Séminaire des Missions Étrangères comptait douze aspirants. Comme la Société n'avait pas encore de maison à sa disposition pour dispenser les études théologiques, les aspirants avaient été placés dans les Grands-Séminaires de Québec et de Montréal. La première ordination sacerdotale eut lieu le 6 mai 1923. Il s'agissait de l'abbé Joseph Roberge qui en était à sa troisième année de Grand-Séminaire quand il avait choisi d'entrer dans la Société ${ }^{30}$. En septembre 1924 le séminaire ouvrait officiellement ses portes. La Société y regroupa ses quinze séminaristes et l'enseignement théologique fut donné par les sept prêtres qui étaient à ce moment-là agrégés au séminaire.

Le 11 septembre 1925, les abbés Lapierre, Bérichon et Lomme partaient pour la Mandchourie. La cérémonie du départ eut lieu au séminaire sous la présidence de $\mathbf{M}^{\mathrm{sr}}$ Forbes ${ }^{31}$. Les premiers efforts donnaient leurs résultats. Avec les années, les vocations se feraient de

27 ASMEQ, Autour du Séminaire canadien des Missions Etrangères.

28 ASMEQ, AJS.

29 ASMEQ, CSFX, 89.

30 Ibid., 143.

31 Ibid., 225-228. 
plus en plus nombreuses. En 1930, la Société comptait trente-trois sémi. naristes et vingt-sept prêtres. Les plus grands espoirs étaient permis ${ }^{32}$.

\section{3 - Reconnaissance civile et canonique}

Parallèlement aux efforts consacrés à l'organisation matérielle et au recrutement des vocations, des démarches étaient faites pour donner à la Société son statut civil et canonique. Cette tâche était confiée au chanoine Gignac dont le nom figurait parmi ceux des premiers collabora. teurs de l'œuvre ${ }^{33}$. En mai 1921, avec l'aide d'un juriste distingué, le juge Adjutor Rivard, il commençait la préparation du projet de loi qui serait soumis à la Législature provinciale pour constituer en corporation la Société des Missions Étrangères. Le 12 septembre, il remettait à $\mathbf{M}^{\mathrm{gr}}$ Forbes une copie du projet de loi et les formules d'incorporation, en recommandant d'obtenir l'existence corporative par Acte de la Législature plutôt que par Lettres patentes. L'incorporation par Acte de la Législature était moins expéditive et elle coûtait moins cher, mais elle n'avait pas cette obligation que comportait l'incorporation par Lettres patentes de faire un rapport annuel au Secrétaire de la Province et de déposer les règlements généraux de la Société. Le chanoine Gignac avait aussi modifié le nom sous lequel le comité d'organisation du séminaire voulait faire incorporer la Société. Il suggérait de remplacer le terme Séminaire Saint-François-Xavier par Société canadienne-française des missions. Les raisons de ce changement étaient que le nom de SaintFrançois-Xavier désignait déjà plusieurs autres corporations et que le mot "séminaire " ne signifiait qu'une partie de la réalité que serait la Société des Missions Étrangères ${ }^{34}$. A leur réunion du 23 septembre 1921, les évêques optèrent pour l'incorporation par Acte de la Législature et ils choisirent comme nom corporatif de la Société: la Société des Missions Étrangères de la province de Québec ${ }^{35}$.

Par suite, le chanoine Roch et le chanoine Gignac entreprirent les démarches nécessaires pour obtenir l'incorporation. Conformément à la loi, le 15 décembre 1921, la Gazette officielle contenait l'avis que les évêques de la province s'adressaient à la Législature pour obtenir l'adoption d'une loi les constituant en corporation sous le nom de la Société des Missions Etrangères de la Province de Québec ${ }^{36}$. La demande d'incorporation civile fut adressée par M. Cannon, député de Québec-centre, au lieutenant-gouverneur de la province, à l'Assemblée législative et au Conseil législatif. Cette demande avait été signée par tous les évêques ayant juridiction totale ou partielle dans la province. Le parlement adopta le

\footnotetext{
32 ASMEQ, AME, 178.

ASMEQ, $D P V C$, troisième réunion.

ASMEQ, CSFX, 62.63.

35 ASMEQ, $D P V C$, quatrième réunion.

36 ASMEQ, CSFX, 65.
} 
texte de loi qui fut sanctionné, le 8 mars 1922, par Sir Charles Fitzpatrick, lieutenant-gouverneur de la province ${ }^{37}$.

Les évêques avaient demandé cette incorporation afin de « mieux organiser, assurer et diriger la participation des catholiques de la province de Québec à la propagation de la foi catholique romaine ». Le cardinal Bégin, archevêque de Québec, $\mathbf{M}^{\mathrm{gr}}$ Roy, coadjuteur de Québec, $\mathrm{M}^{\mathrm{gr}}$ Emard de Valleyfield, $\mathrm{M}^{\mathrm{gr}}$ Labrecque de Chicoutimi, $\mathrm{M}^{\mathrm{gr}}$ Larocque de Sherbrooke, $\mathbf{M}^{\mathrm{gr}}$ Cloutier de Trois-Rivières, $\mathrm{M}^{\mathrm{gr}}$ Brunault de Nicolet, $M^{\text {gr }}$ Bernard de Saint-Hyacinthe, $M^{\text {gr }}$ Latulippe de Haileybury, $\mathbf{M}^{\mathrm{gr}}$ Ryan de Pembroke, $\mathbf{M}^{\mathrm{gr}}$ Gauthier, administrateur de Montréal, $\mathbf{M}^{\mathrm{gr}}$ Forbes de Joliette, $\mathbf{M}^{\mathrm{gr}}$ O'Leary de Charlottetown, $\mathrm{M}^{\mathrm{gr}}$ Ross, administrateur de Rimouski, signèrent cette demande d'incorporation. Ce projet de loi stipulait que tous les évêques déjà mentionnés ainsi que toutes les autres personnes qui

\begin{abstract}
d'après les règles qui seront établies pour cet objet, leur succéderont ou leur seront adjointes et deviendront membres de la corporation, sont constitués en corporation sous le nom de la Société des Missions Etrangères de la province de Québec avec succession perpétuelle et avec les droits, pouvoirs et privilèges appartenant aux corporations civiles ordinaires.
\end{abstract}

Le but de la corporation était de travailler à la propagation de la foi, de faire contribuer les catholiques de la province à l'évangélisation en terre étrangère, de favoriser le recrutement et la formation des vocations missionnaires. Pour ce, la corporation était autorisée à construire des séminaires, * à percevoir des secours, à faire de la propagande par la parole et les écrits, à publier des bulletins et des revues ». Elle jouissait de tous les pouvoirs accordés aux corporations civiles comme, par exemple, d'acheter des biens mobiliers et immobiliers, les administrer et en retirer des revenus, faire des placements, emprunter, émettre des obligations, etc. Elle était libre de se donner les règlements qu'elle jugeait opportun d'adopter concernant l'administration des biens, le gouvernement de la Société, la nomination des membres, l'organisation du conseil de direction pour autant que ces lois seraient conformes aux lois de la province et de l'Église. Le siège social de la corporation était fixé à Montréal. $\mathbf{M}^{\mathrm{gr}}$ Gauthier, administrateur du diocèse de Montréal, $\mathbf{M}^{\mathrm{gr}}$ Roy, coadjuteur de Québec, $\mathbf{M}^{\mathbf{g r}}$ Forbes, évêque de Joliette, et $\mathbf{M}^{\mathrm{gr}}$ Léonard, évêque de Rimouski, formèrent

un conseil provisoire de direction, chargé d'exercer les pouvoirs et les droits de la corporation jusqu'à ce qu'il soit pourvu autrement en conformité des règlements qui seront adoptés ${ }^{38}$.

Deux ans après que la Société eut obtenu sa charte civile, des négo. ciations commencèrent pour obtenir de Rome l'érection canonique de la Société et du séminaire. Comme le siège social de la corporation était à

37 ASMEQ, $D P V C$, cinquième réunion.

38 ASMEQ, $D D R F$, texte du projet de loi. 
Montréal, le Conseil d'administration du séminaire chargea l'administration apostolique de ce diocèse de faire les démarches relatives à cette fin ${ }^{39}$. $\mathrm{M}^{\mathrm{gr}}$ Gauthier demanda donc au Souverain Pontife la permission " d'émettre un décret d'érection de la dite Société en Congrégation cléricale, selon les saints Canons ». Celui-ci appuyait sa demande sur le fait que la Société avait déjà reçu l'approbation de la Propagande, qu'elle avait sa charte civile et qu'elle était constituée en corporation. De plus, elle possédait son séminaire et elle comptait déjà cinq prêtres et douze aspirants ${ }^{40}$. La Congrégation de la Propagande agréa cette demande et, le 6 décembre 1924, elle chargea $\mathrm{M}^{\mathrm{gr}}$ Gauthier, à titre d'Ordinaire du lieu où la Société existait au point de vue civil et religieux, d'émettre le décret d'érection de la Société en Congrégation cléricale et d'en approuver les règlements provisoires. Le 6 janvier 1925, il émettait le décret d'érection de la Société.

En vertu des pouvoirs accordés nous établissons canoniquement la Société des Missions Etrangères de la Province de Québec. En vertu des présentes, nous désignons le Séminaire des Missions Etrangères de la Province de Québec, que cette Société a fondé en notre diocèse, sous le vocable et la protection de Saint-François-Xavier, patron des missions et patron secondaire du Canada, comme le noviciat régulier où se forment nos futurs missionnaires, et nous le déclarons à ce titre canoniquement désigné, avec tous les droits, grâces et privilèges dont jouissent les autres maisons de noviciat ainsi désignés par autorité apostolique. Nous permettons aux jeunes clercs qui voudront se consacrer aux missions, dans la Société des Missions Etrangères de la Province de Québec, de faire à l'époque du sous-diaconat, le serment de stabilité selon l'article 55 des règlements de la Société.

Il procéda ensuite à l'érection canonique du séminaire sous le vocable de Saint-François-Xavier, il en approuva les règlements provisoires et il lui accorda les privilèges dont jouissaient les autres séminaires de missions ${ }^{41}$.

\section{4- Gouvernement interne}

Entre-temps, le chanoine Gignac avait commencé la préparation des constitutions canoniques en se servant des constitutions des Pères Blancs, que la Congrégation de la Propagande avait envoyées comme modèle ${ }^{42}$. L'assemblée des évêques approuvait, le 10 mai 1922, les règlements provisoires de la Société ${ }^{43}$. En janvier $1925, \mathrm{M}^{\mathrm{gr}}$ Gauthier émettait le décret d'érection canonique de la Société et du Séminaire. La Société était donc en mesure de lier ses premiers membres par un serment temporaire de trois ans. Ce fut $\mathrm{M}^{\mathrm{gr}}$ Forbes qui reçut le serment de stabilité du chanoine Roch et des abbés Lapierre, Rondeau, Geoffroy, Roberge, Bérichon, Chaumont et de six séminaristes, le 2 juin $1925^{44}$.

39 ASMEQ, $A M E, 75,5$ février 1924, douzième réunion.

40 Ibid., 80-81, 21 février 1924, Gauthier au Pape.

41 ASMEQ, DDRF, décret d'érection.

42 ASMEQ, CSFX, 13 mars 1922, Van Rossum à Forbes.

43 ASMEQ, DPVC, sixième réunion.

14 ASMEQ, CSFX, 212. 
Afin de hâter l'approbation des constitutions, le chanoine Roch partit pour Rome, le 18 octobre 1927. Le 2 novembre, il remettait les constitutions au cardinal Van Rossum, préfet de la Propagande. Le lendemain, $\mathrm{M}^{\mathrm{kr}}$ Pecorari, sous-secrétaire de la Propagande, les lui rendait avec le sceau de la Propagande.

A la dix-neuvième session du Conseil d'administration, le chanoine Roch demanda s'il ne vaudrait pas mieux remettre le gouvernement général de la Société et du séminaire aux membres de la Société. Le Conseil d'administration accepta de former un conseil général pour le gouvernement interne de la Société et du Séminaire, mais il conserva le statu quo touchant l'administration civile et financière. $\mathrm{M}^{\mathrm{gr}}$ Gauthier qui avait érigé canoniquement la Société et le Séminaire a nommé, en cette réunion,

M. le chanoine J. A. Roch, Supérieur Génèrai de İa Société et du Séminaire, assisté de quatre conseillers, les abbés Chaumont, premier assistant et vice-supérieur, J. Roberge, second assistant et vice-directeur du Séminaire, C. Rondeau, économe du Séminaire, J. Geoffroy, directeur du Séminaire et secrétaire général. Le conseil exercera ses fonctions en conformité avec le droit canonique et les règlements provisoires de la Société $\mathbf{4 5}$.

Le Supérieur général prononça son serment perpétuel de stabilité à la Société, le $1^{\text {er }}$ juin 1928, en présence de l'abbé Chaumont, délégué par $\mathrm{M}^{\mathrm{kr}}$ Gauthier. Le Supérieur général reçut ensuite le serment perpétuel des autres membres de la Société. Une cérémonie semblable se déroula, le 4 juin, en Mandchourie, alors que l'abbé Lapierre prêta serment et reçut le serment perpétuel des sept autres missionnaires de la Société ${ }^{48}$.

Rome émettait le décret pontifical approuvant pour sept ans les constitutions de la Société des Missions Étrangères, le 25 juillet $1929^{47}$. Les constitutions définissaient la Société des Missions Étrangères comme:

un institut de clercs séculiers vivant en communauté, pratiquant la même règle, et liés entre eux et à l'œuvre commune par le serment de se consacrer aux missions de la Société, selon les constitutions et sous l'obéissance du supérieur légitime.

Le but de la Société était la sanctification de ses membres et l'œuvre des missions en terre païenne, par l'évangélisation des infidèles. Les prêtres de la Société étaient sous la juridiction des Ordinaires des diocèses pour le culte et le ministère. Quant à l'observance des Constitutions, ils relevaient de leur Supérieur général. L'institut pouvait s'adjoindre des auxiliaires laïcs. Les évêques de la province demeuraient les protecteurs de la Société.

45 ASMEQ, $A M E, 144-145,8$ mai 1928, dix-neuvième réunion.

46 Ibid., 146-147.

47 ASMEQ, CSFX, 269. 
Lo Chapitre général de la Société était sous la juridiction de la Propagande, l'autorité suprême de l'institut pour tous ses intérêts spirituels et temporels. Il avait pour fonction de nommer le Supérieur général et ses assistants et de régler les questions majeures qui dépassaient la compétence du Supérieur général en conseil. Le Supérieur général, assisté de son conseil, voyait au gouvernement de la Société concernant les nominations, l'admission aux serments et aux ordres, l'approbation des règlements des coutumiers généraux et particuliers, l'achat et la vente d'immeubles, l'érection des circonscriptions, l'acceptation de nouvelles missions, etc. Les établissements de la Société étaient groupés en circonscriptions qui avaient à leur tête un supérieur régional assisté d'un conseil.

La Société se composait de missionnaires, prêtres et séminaristes par un serment temporaire ou perpétuel. Avant de devenir membre de la Société, l'aspirant devait suivre pendant un an le règlement du séminaire central, après quoi il pouvait être autorisé à faire un serment temporaire de trois ans et puis un serment perpétuel. Le serment fait devant la Société n'avait pas la valeur des vœux religieux. Trois obligations découlaient du serment, à savoir la stabilité dans la Société, le dévouement à l'œuvre et l'obéissance aux supérieurs. Los membres de la Société ne recevaient pas de rémunérations temporelles pour leurs services dans la Société. Les biens reçus en leur qualité de missionnaires revenaient à la Société ou à la mission pour laquelle ils avaient été donnés. Ils conservaient cependant les dons qui leur étaient faits personnellement ainsi que les honoraires de messes. La Société pourvoyait à l'entretien de ses membres, mais les dépenses personnelles comme les vêtements, les livres, demeuraient à la charge des membres.

Concernant les règles de vie apostolique, les constitutions définissaient que la principale activité des missionnaires était la prédication de l'Évangile. Cependant, les missionnaires devaient par les œuvres de charité corporelle, telles l'établissement d'écoles, de dispensaires et d'orphelinats, amener les gens à la foi. De même, ils devaient former des catéchistes pour se faire aider dans leur travail, établir un clergé indigène, cultiver les vocations. Les adultes ne devaient pas être admis au baptême sans avoir subi un sérieux cathécuménat. Les missionnaires avaient droit à la vie commune. Ils ne devaient jamais être moins de deux et, quand les circonstances le permettaient, ils devaient faire les exercices religieux et prendre les repas du midi et du soir en commun ${ }^{48}$.

La Société avait définitivement en main son gouvernement interne. Elle s'administrait par elle-même selon ses constitutions. Quant à l'admi-

48 ASMEQ, Constitutions de la Société des Missions Etrangères de la Province de Québec. 
nistration financière et civile, la Société dépendait encore du Conseil d'administration formé de quatre évêques et de trois de ses membres.

\section{5. - Demande d'un territoire de mission}

Les évêques souhaitaient que la Chine devienne la terre de missions où les prêtres de la nouvelle Société canadienne porteraient l'Évangile. Tel fut le vœu que $\mathrm{M}^{\mathrm{gr}}$ Forbes transmit au cardinal Van Rossum, préfet de la Propagande, en lui communiquant la décision des évêques de fonder un Séminaire des Missions Etrangères dans la province de Québec. La grande détresse spirituelle de ce pays et les espoirs nouveaux qui se présentaient pour l'évangélisation de ce peuple étaient à l'origine de ce désir de l'épiscopat ${ }^{49}$. Le 2 février 1922, $\mathrm{M}^{\mathrm{gr}}$ Forbes rappela de nouveau au préfet de la Propagande le désir des évêques d'avoir un territoire en Chine comme première terre d'évangélisation pour leurs missionnaires ${ }^{50}$. Le cardinal Van Rossum répondit que la Propagande était favorable à ce que la Société puisse envoyer ses missionnaires en Chine, mais qu'elle ne lui confierait un territoire que quand elle serait en mesure d'en assumer les responsabilités ${ }^{51}$. La Société entreprit donc, à la suite de cette réponse, de préparer ses sujets en fonction de la Chine ${ }^{52}$.

Pendant ce temps, le séminaire se développait et déjà, en 1924, il comptait un bon nombre d'aspirants. Cette nouvelle ouvre ne manquait pas d'intéresser certains évêques de missions qui espéraient trouver là une bonne source de vocations pour leur prêter secours. La première offre d'un territoire de mission parvint à la Société le 18 janvier 1924. Elle venait de $\mathrm{M}^{\mathrm{gr}}$ Cothonay, préfet apostolique en Chine, le même qui en 1899 avait fait des démarches pour obtenir l'établissement d'un séminaire des Missions Étrangères dans la province de Québec. La Société répondit à $\mathrm{M}^{\mathrm{sr}}$ Cothonay qu'il revenait à la Congrégation de la Propagande de lui assigner un territoire et que, par conséquent, elle ne pouvait rien promettre ${ }^{53}$.

Une autre offre de territoire fut transmise à la Société, le 5 mars de la même année. L'archevêque de Pondichery, en Inde, offrait une section de son territoire dont une grande partie ne pouvait être évangélisée faute de missionnaires ${ }^{54}$. La Société exprima ses regrets à $\mathrm{M}^{\mathrm{gr}}$ Morel, archevêque de Pondichery, de ne pouvoir répondre à sa demande, étant donné que le Séminaire avait été fondé pour la Chine ${ }^{55}$.

Le 31 mars, une troisième démarche était entreprise pour obtenir l'aide de la Société. La demande fut formulée par $\mathbf{M}^{\mathrm{rr}}$ Blois, vicaire

49 ASMEQ, $A M E, 14,24$ février 1921, Forbes à Van Rossum.

so Ibid, 31 .

51 Ibid., 32.

52 Ibid., 34.

ธ3 ASMEQ, CSFX, 155.

54 Ibid., 155-156.

55 Ibid., 156. 
apostolique de Moukden en Mandchourie. Il proposait à la Société d'étu. dier la possibilité d'envoyer ses missionnaires en Mandchourie, si aucune décision n'avait encore été arrêtée par la Propagande sur le choix d'un territoire. Cette contrée comprenait six résidences de missionnaires et environ vingt-cinq mille chrétiens connus et plusieurs autres dispersés dans des régions éloignées des centres. Le centre de cette contrée était la ville de Tao-Nan qui était reliée à la capitale de la province de Moukden par une ligne de chemin de fer. En plus de ce territoire, la Société pourrait acquérir la province de Tsitsikar au nord, car $\mathrm{M}^{\mathrm{gr}}$ Gapais était disposé à céder cette partie de son vicariat.

La Société avait, selon $\mathrm{M}^{\mathrm{gr}}$ Blois, de grands avantages à accepter la Mandchourie comme terre de missions, car le climat était comparable à celui du Canada, et on pouvait y faire pousser les mêmes cultures. De plus, il y avait une population homogène, parlant une même langue et ayant des coutumes semblables. Le pays était immense et de nombreux vicariats y seraient érigés avec le temps. Les forces de la Société seraient pendant longtemps absorbées dans ce pays. La Société éviterait donc la dispersion de ses membres dans des régions différentes où la langue et les coutumes n'étaient pas les mêmes. Ceci avait pour intérêt que, lors. qu'il y aurait plusieurs vicariats dans le pays, les supérieurs ecclésiastiques à la tête de ces territoires seraient membres d'une même Société, ils seraient aussi de même nationalité et auraient été formés à la même école, ce qui favoriserait une meilleure collaboration entre les vicariats. Il arrivait aussi que certaines missions étaient incapables de fournir des hommes aptes à assumer des fonctions d'autorité et qu'il fallait les pren. dre ailleurs. Quand les missionnaires étaient dispersés sur des territoires variés où la langue et les coutumes étaient différents, il devenait difficile de transférer les missionnaires d'un poste à l'autre, car ils devaient prendre des années pour se familiariser avec la langue et les mours de leurs nouvelles missions. En venant en Mandchourie, la Société éviterait tous les inconvénients de la dispersion qui avait posé quantité de problèmes à d'autres communautés religieuses. $M^{\text {gr }}$ Blois faisait cette démarche avec l'approbation de $\mathrm{M}^{\mathrm{gr}}$ Gapais, vicaire apostolique de la Mandchourie septentrionale, et avec l'approbation du délégué apostolique de la Chine ${ }^{56}$.

Le Comité d'organisation du séminaire envoya une copie de cette lettre à tous les évêques de la province, leur demandant de donner leur avis sur cette proposition ${ }^{57}$. Presque tous les évêques se montrèrent favorables à cette offre et ils souhaitèrent qu'une demande soit soumise au préfet de la Propagande pour obtenir ce territoire de missions ${ }^{58}$. $\mathrm{M}^{\mathrm{gr}}$ Blois

56 ASMEQ, AME, 83.

57 Ibid., 82, 13 mai 1924.

58 Ibid., 89-90, 23 juin 1924. 
fut aussi informé que son offre avait été très bien accueillie par l'ensemble des évêques, mais que la Société ne pouvait lui donner de réponse définitive avant d'avoir eu l'agrément de la Congrégation de la Propagande ${ }^{59}$.

Les premiers prêtres de la Société allaient bientôt pouvoir partir pour les missions, mais aucun territoire ne leur avait encore été assigné. $\mathrm{M}^{\mathrm{gr}}$ Gauthier avait pourtant présenté une demande de territoire à la Propagande lors de son passage à Rome, et $\mathrm{M}^{\mathrm{gr}}$ Blois avait aussi exprimé à la Propagande son désir de voir venir les missionnaires canadiens en Mandchourie, mais aucune réponse n'avait été donnée ${ }^{60}$. Le Comité d'organisation jugea bon d'insister et, le 23 février 1925, $\mathbf{M}^{\mathrm{gr}}$ Forbes, au nom de l'administration de la Société et de l'assemblée des évêques, écrivit au cardinal Van Rossum pour lui demander :

de permettre que soient dirigés vers le vicariat apostolique de la Mandchourie méridionale les trois de nos premiers missionnaires qui après quatre années passées sous la direction du supérieur de notre Séminaire Saint-François-Xavier au Pont-Viau seront prêts dès le mois de juillet prochain à partir pour le champ d'apostolat.

$\mathrm{M}^{\mathrm{gr}}$ Forbes lui rappela le désir de $\mathrm{M}^{\mathrm{gr}}$ Blois de voir les missionnaires canadiens collaborer avec lui, ainsi que l'acceptation des évêques du Québec de voir la Société se dévouer dans le vicariat apostolique de Moukden jusqu'à ce que les Canadiens soient assez nombreux pour accepter la responsabilité d'un territoire déterminé que la Propagande voudrait bien leur confier. Ce serait aussi un avantage pour le recrutement des vocations que de savoir où les missionnaires exerceraient leur apostolat ${ }^{61}$. Le 9 mai 1925, le cardinal Van Rossum autorisa la Société à envoyer ses missionnaires en Mandchourie. Les missionnaires seraient placés sous la juridiction de $\mathrm{M}^{\mathrm{gr}}$ Blois, vicaire apostolique de Moukden.

Dès qu'ils seront rendus dans les missions, ils chercheront à apprendre la langue et les coutumes des lieux sous la sage direction de $\mathrm{M}^{\mathrm{gr}}$ Blois, et quand ils auront acquis l'expérience voulue, il leur déterminera le territoire où ils devront exercer leur ministère. ${ }^{62}$

Cette bonne nouvelle fut aussitôt transmise à $\mathrm{M}^{\mathrm{gr}}$ Blois qui suggéra à la Société d'entreprendre immédiatement les démarches nécessaires auprès de la Propagande, pour se faire réserver, en plus de la partie de son vicariat qu'il allait lui céder, la vaste province de Tsitsikar, afin qu'elle puisse avoir un territoire assez grand pour se développer sans avoir à subir les inconvénients de l'éparpillement de ses membres. Si cette demande était faite à temps, elle avait des chances de réussir, d'abord parce que ce territoire, dont voulait se départir $\mathrm{M}^{\mathrm{gr}}$ Gapais, n'avait encore été attribué à

$59 \quad$ Ibid., 88, $1^{\text {er }}$ juillet 1924.

60 Ibid., 99.

61 Ibid., 103-104.

62 Ibid., 123. 
aucune communauté et aussi parce que la Propagande conviendrait du bien-fondé de cette demande ${ }^{63}$.

\section{6. - Arrivée des missionnaires en Mandchourie}

La Mandchourie se situait dans l'Asie Orientale, au nord de la Chine. Ce pays avait été jusque-là évangélisé par les prêtres des Missions Étrangères de Paris. Ils avaient rencontré beaucoup de difficultés. En 1900, l'insurrection des Boxers avait fait de nombreuses victimes parmi les chrétiens. $\mathbf{M}^{\mathrm{gr}}$ Guillen, neuf de ses prêtres et des centaines de chrétiens avaient été massacrés. Leurs œuvres étaient constamment menacées par les révolutions et les brigandages. En 1923, la population catholique de Mandchourie comptait 57,122 âmes. On trouvait dans le vicariat de Moukden un grand et un petit séminaire pour la formation des indigènes qui songeaient au sacerdoce. Il existait des écoles chrétiennes qui dispensaient l'instruction à plus de trois mille enfants des orphelinats et des hospices qui abritaient plus de mille vieillards et enfants. La population du vicariat de Moukden comptait environ douze millions de population et pour s'occuper de l'évangélisation et des œuvres, $M^{\text {gr }}$ Blois n'avait que vingt-quatre missionnaires européens, vingt-deux prêtres chinois, une vingtaine de religieuses indigènes ${ }^{64}$.

Telle était la situation quand les trois premiers missionnaires de la Société débarquèrent sur la terre de Chine après un voyage de plus d'un mois. L'abbé Lomme fut hospitalisé à Shanghai dès son arrivée, le 17 octobre 1925, et il allait demeurer au lit jusqu'en mai 1926. Les abbés Bérichon et Lapierre se rendirent à Moukden. L'abbé Lapierre qui ne se sentait pas bien depuis son débarquement à Newchuang dut être hospitalisé quelques mois après son arrivée. Sa maladie était le paludisme; après un traitement d'une quinzaine de jours, il était de nouveau sur pied et il quittait l'hôpital de Shanghai pour revenir à Moukden ${ }^{65}$.

Quel genre de vie attendait les missionnaires canadiens à leur arrivée ? $\mathrm{M}^{\mathrm{gr}}$ Blois faisait remarquer qu'il aurait été imprudent de les laisser à eux-mêmes, car, ne connaissant pas la langue, les coutumes et la mentalité des indigènes, ils se seraient vite empêtrés dans des difficultés de toutes sortes. Tous les jeunes missionnaires qui arrivaient en mission étaient logés à la maison centrale des missions ou auprès d'un missionnaire expérimenté jusqu'à ce qu'ils aient une connaissance suffisante de la langue et des usages pour pouvoir se débrouiller seuls. Ils n'était donc pas question de donner immédiatement la direction d'un territoire de missions aux premiers missionnaires canadiens. Ils resteraient sous la juridiction du vicaire apostolique de Moukden pendant un délai d'au moins cinq ans. Le succès de leur entreprise pourrait être considérable-

63 Ibid., 113.

64 ASMEQ, AJS, 3 septembre 1926, Geoffroy au Devoir.

65 ASMEQ, CSFX, 234, Bérichon à Roch. 
ment compromis par les difficultés énormes et les erreurs de l'inexpérience s'ils voulaient devenir trop tôt autonomes.

Les trois missionnaires se mirent donc à l'étude de la langue et des mours des gens du pays. Pour apprendre la langue, le jeune missionnaire était confié à un professeur indigène qui pouvait, mieux qu'un étranger n'aurait pu le faire, donner la vraie prononciation. Cependant, la présence d'un missionnaire expérimenté était aussi indispensable pour l'aider à résoudre certaines difficultés que l'indigène ne soupçonnait même pas. Pour l'étude et l'intelligence des coutumes du pays, de la mentalité des indigènes et des méthodes d'apostolat, il était encore bien plus nécessaire de profiter de l'expérience acquise et des traditions établies, au prix de bien des erreurs et des faux pas ${ }^{66}$.

Les missionnaires canadiens apprirent donc leur métier auprès d'anciens missionnaires qui les firent profiter de leur expérience. Dans une lettre envoyée au supérieur de Pont-Viau en janvier 1926, $\mathrm{M}^{\mathrm{gr}}$ Blois disait :

Les Pères Lapierre et Bérichon n'ont pas attendu M. Lomme pour se livrer à l'étude du chinois. Ils s'y sont mis avec beaucoup de courage et non moins de succès. Le Père Bérichon commence de petites conversations avec nos prêtres indigènes. Il $\mathrm{y}$ met tant d'ardeur qu'il arrivera vite à se tirer d'affaire.

Quant aux Pères Lapierre et Lomme, la maladie retarda leur progrès, mais ils allaient vite reprendre le temps perdu ${ }^{67}$.

Ce stade de formation était indispensable pour assurer le succès de l'œuvre. Tous les missionnaires devaient franchir cette étape avant d'entrer dans le feu de l'action.

\section{7 - Premières missions et méthodes d'apostolat}

La région où les missionnaires canadiens allaient entreprendre leur activité apostolique avait une étendue d'environ huit cents milles de longueur sur cent quatre-vingts de largeur. Elle était partagée en deux parties très distinctes, le sud et le nord. La population du sud était assez dense. On y trouvait de grandes villes. Cette partie était traversée par deux lignes de chemin de fer. Les terres étaient en général assez cultivables. Les catholiques y étaient peu nombreux et il n'y avait que cinq postes avec missionnaires résidents. Par contre, le nord était peu peuplé et on ne trouvait que quelques villages importants. Chaque année des milliers d'immigrants venaient s'installer dans cette région. Le pays était plat dans presque toute son étendue et absolument déboisé. Il y avait quelques bonnes terres cultivables, mais la majeure partie était faite de sable et de terrain salpêtre. La contrée du nord était plus que toute autre la proie du brigandage. Pour se défendre, les colons se groupaient en de

66 Ibid., 217.221.

67 Ibid., 239. 
petits villages entourés de hauts murs en terre. Mais ce système de défense devenait désuet, car les brigands possédaient des armes modernes. Un millier de chrétiens y vivaient dispersés. La plupart étaient des immigrants qui avaient quitté leur province parce qu'ils n'y trouvaient plus de quoi vivre. Aucun missionnaire ne résidait dans cette contrée. De temps à autre, un missionnaire de Moukden venait visiter les principaux groupes de chrétiens ${ }^{68}$.

En 1927, le père Lapierre se rendait à Taonan, ville centrale de la partie nord du territoire, pour y construire une résidence. Le père Bérichon devenait curé de Maimaikai et le père Lomme, vicaire à Lipeng. Après avoir passé leur examen de juridiction, les sept autres missionnaires canadiens, qui étaient arrivés en Mandchourie en 1926, recevaient leur nomination. Le père Barbeau était nommé auxiliaire du père Bérichon à Maimaikai. Le père Larochelle recevait la cure de Fagu, le père Forcier allait occuper le poste de Kangping, le père Paradis partait comme vicaire à Gaochantoun et le père Charest à Nieuchwang. Quant aux pères Jasmin et Berger, ils allaient aider le père Lapierre à Taonan. Ce petit groupe de missionnaires canadiens avait le père Lapierre comme supérieur, assisté des pères Bérichon, Larochelle, Lomme et Jasmin ${ }^{69}$.

Au cours de ces premières années d'apostolat, les missionnaires canadiens partirent à la conquête du territoire qui leur était assigné, en occupant les postes de missions déjà existants et en établissant de nouveaux postes là ou les besoins étaient plus urgents. En arrivant dans sa mission, le missionnaire voyait d'abord à se trouver un logement, à y organiser une chapelle et à prendre contact avec les chrétiens qui pouvaient se trouver sur son territoire. Suivant les exigences du milieu et selon ses possibilités, chaque missionnaire voyait à organiser sa chrétienté et à évangéliser sa contrée. Pour amener les gens à la foi et même pour affermir les chrétiens déjà existants, quatre moyens avaient paru particulièrement importants aux missionnaires, qui tâchèrent de les réaliser dans la mesure du possible et là où leur importance se faisait principalement sentir.

Le catéchiste était d'un grand secours au missionnaire. Son rôle consistait à rassembler les fidèles dispersés dans la région, à les instruire et à voir quels étaient leurs besoins. Il informait ensuite le missionnaire de tout ce qui était utile pour son ministère. Il présidait à la prière des chrétiens à la chapelle. Il enseignait le catéchisme à ceux qui étaient inscrits au catéchuménat. En fait, il préparait le terrain à la venue du missionnaire qui, bien renseigné, n'avait pas à tâtonner à son arrivée dans la mission. Le catéchiste était moins nécessaire dans certains régions, mais il devenait indispensable dans d'autres comme la mission du père Berger

68 ASMEQ, AJS, 127, Berger au Devoir.

69 ASMEQ, $A M E$, 16 mai 1927. 
à Hwaite. Bref, il allégeait le fardeau du missionnaire qui avait à s'occuper du ministère auprès des fidèles, de l'administration de la mission, du bon fonctionnement des écoles. De plus, le missionnaire à ses débuts n'a. vait pas une connaissance suffisante de la langue pour faire comprendre le catéchisme aux jeunes et, à ce moment-là, le catéchiste lui était très utile ${ }^{70}$.

L'École apparaissait aussi comme un moyen indispensable au missionnaire pour l'efficacité de son action. En Chine, le nombre des illettrés était considérable et les écoles étaient insuffisantes pour recevoir tous les enfants qui étaient en âge de les fréquenter. Les programmes mis en vigueur dans les écoles publiques étaient à base d'athéisme. Dans le district où se trouvaient les missionnaires canadiens, les écoles publiques étaient en nombre insuffisant; aussi, dès qu'il arrivait dans une mission, le missionnaire n'avait rien de plus à cour que d'ouvrir une école et d'y attirer les enfants des familles chrétiennes et aussi des familles païennes ${ }^{71}$. Dans ces écoles, on enseignait le catéchisme et quel. ques éléments de connaissance profane, car les parents tenaient à ce que les enfants aient une certaine dose de savoir. Si l'école catholique n'avait pas enseigné les lettres, les enfants seraient allés à l'école publique ${ }^{72}$. Évidemment ces écoles étaient un coûteux fardeau pour le missionnaire, car les parents ne voulaient souvent pas payer pour envoyer leurs enfants à l'école de la mission; mais c'était là un moyen presque indispensable pour donner l'instruction religieuse et les efforts nécessaires y furent consacré ${ }^{73}$.

L'œuvre des dispensaires apparaissait comme le meilleur moyen d'entrer en relation avec les païens. A la mission de Leao Yuan, par exemple, les malades venaient par centaines au dispensaire. Un catéchiste avait été affecté à ce dispensaire pour apprendre aux visiteurs les premiers éléments de la doctrine chrétienne. Les religieuses avaient habituellement la responsabilité de ces dispensaires et leur dévouement gratuit auprès des malheureux en faisant des témoins privilégiés de la foi, car les païens, qui comprenaient difficilement que l'on puisse se dé. vouer gratuitement, finiraient par comprendre qu'il y avait quelque chose de plus grand que l'argent. Les religieuses qui étaient appelées au chevet des malades en profitaient pour faire des baptêmes d'enfants et d'adultes. Les dispensaires favorisaient les inscriptions au catéchuménat et les missionnaires canadiens en établissaient dans leurs missions dès que cela était possible.

Une autre œuvre importante à laquelle les missionnaires consacrèrent particulièrement leurs efforts fut celle des immigrants. La région du

70 ASMEQ, AJS, Pradis au Devoir.

71 ASMEQ, SSME, 110-116.

72 ASMEQ, AJS, Paradis au Devoir.

73 Ibid. 
nord offrait de grands avantages à la colonisation et des milliers de colons arrivaient à chaque année pour s'y établir. Parmi ces immigrants, il y avait des chrétiens et le missionnaire cherchait à les rassembler. Ces chrétiens, qui étaient souvent peu formés, couraient de graves dangers à rester isolés parmi les païens; il fallait donc les regrouper pour pouvoir leur donner une meilleure instruction religieuse. Cependant, ces nouveaux arrivés voulaient aussi posséder des terres. A Toutsuen, par exemple, il y avait quelques terres fertiles, mais il y en avait beaucoup qui étaient impropres à la culture. Le père Berger, qui était à la tête de cette mission, décida de leur venir en aide, car ces immigrants étaient plutôt pauvres, et ils ne pouvaient par conséquent acheter des terres fertiles. Établis sur des terres qui leur auraient peu rapporté, ils auraient de nouveau changé de pays après deux ou trois ans. Le père Berger acheta donc, avec l'aide de quelques confrères, plusieurs bonnes terres qui furent divisées et vendues aux chrétiens dans des conditions réalisables. Ceux-ci formèrent donc un petit village qui fut mis sous la protection du Sacré-Cœur. Les récoltes furent bonnes et l'avenir de ce village semblait assuré. Une école y fut construite ainsi qu'une chapelle. Les familles qui trouvaient là de quoi vivre convenablement ne songeraient sans doute pas à partir. Le problème des immigrants s'accentuait avec les années et l'expérience du père Berger, qui avait donné jusque-là de bons résultats, encourageait à grouper d'autres immigrants chrétiens sur des terres, car c'était le meilleur moyen de préserver leur foi et d'assurer leur pratique religieuse ${ }^{74}$.

\section{8 - Organisation d'une préfecture apostolique}

Pendant que les missionnaires canadiens, qui étaient au nombre de seize en 1928, travaillaient à l'évangélisation de leur territoire en ouvrant de nouveaux postes de missions et en y établissant des écoles et des dispensaires, le Conseil d'administration de la Société entreprenait les démarches nécessaires pour obtenir la division officielle du territoire qui devait revenir à la Société des Missions Étrangères de Québec. Le vicaire apostolique de Moukden fut sollicité par le chanoine Roch d'entreprendre, de concert avec la Société, des négociations avec la Propagande pour obtenir la division du territoire réservé aux missionnaires canadiens. $\mathbf{M}^{\mathrm{gr}}$ Blois répondit qu'il avait déjà posé

les premiers jalons de cette division en nommant, le 8 novembre, le Père Lapierre, vicaire forain pour tout le territoire du Tao Chang Tao. Il aura ainsi occasion de se mêler directement, sans en porter la responsabilité, à certains menus détails d'administration qui ne sont pas sans importance 75 .

Cependant, selon les règlements de la Société des Missions Étrangères de Paris à qui appartenait le territoire, il revenait au Supérieur général

74 ASMEQ, SSME, 116.

75 ASMEQ, $A M E, 152$. 
de cette Société d'engager ce genre de négociations en cour de Rome. $\mathrm{M}^{\mathrm{gr}}$ Blois informait donc le chanoine Roch qu'il avait transféré cette demande au supérieur de Paris ainsi que les renseignements dont il aurait besoin pour traiter cette affaire. Il l'invitait aussi à engager les négociations avec le vicaire apostolique de Jehol de qui dépendait une partie du territoire qui devait revenir à la Société. Cette partie du territoire n'était pas sous la responsabilité des Missions Étrangères de Paris ${ }^{76}$. Le chanoine Roch, qui avait aussi fait une demande à la Congrégation de la Propagande, reçut de celle-ci un long questionnaire que le père Lapierre fut chargé de remplir ${ }^{77}$.

Le 4 avril 1929, le Conseil d'administration de la Société avait en main une lettre de $\mathrm{M}^{\mathrm{gr}}$ Blois qui avait été autorisé par les Missions Étrangères de Paris à céder aux missionnaires canadiens la partie du territoire convenue ${ }^{78}$. Il avait aussi une lettre de $\mathrm{M}^{\mathrm{gr}}$ Abels, vicaire apostolique de Jehol, qui avait obtenu le consentement de la Congrégation du Cour-Immaculée-de-Marie de Scheut, à qui appartenait l'autre partie du territoire, de céder à la Société canadienne « la partie du vicariat de Jehol, qui se trouve dans le Tao Tchang Tao, ainsi que la souspréfecture de Tchang Au Shien, qui au civil dépend de la province de Moukden ${ }^{79}$. A la suite de ces deux autorisations, le Conseil d'administration autorisa le chanoine Roch à faire à la Propagande une demande officielle d'un territoire ${ }^{80}$.

La Congrégation de la Propagande érigea la préfecture apostolique de Szépingkai, avec la ville de Szépingkai comme siège de la préfecture, le 9 juillet $1929^{81}$. Située à la tête d'un chemin de fer, la ville de Szépingkai était un grand centre d'approvisionnement en articles étrangers par le sud et en matériaux de construction par le nord. Il y avait sur place une centaine de chrétiens dont aucun n'était originaire de l'endroit. Des difficultés énormes avaient dû être surmontées pour l'établissement de ce poste. En effet, malgré des interdictions sévères, un prêtre indigène déguisé en marchand avait réussi à acheter un terrain assez vaste pour y installer toutes les ouvres d'un poste central. Ces précautions avaient dû être prises, car un vendeur avait été mis en prison pour avoir voulu céder un terrain à la mission catholique ${ }^{82}$.

Le dimanche 18 août 1929, le père Lapierre, en présence des pères Larochelle, Turcotte et Bérichon et d'une quarantaine de chrétiens, bénissait solennellement la nouvelle église de Szépingkai. Cette église allait

76 ASMEQ, CSFX, 294.

77 ASMEQ, $A M E, 153$.

78 Ibid., 159.

79 ASMEQ, CSFX, 295.

80 ASMEQ, $A M E, 159$.

81 ASMEQ, $A M E, 170-171$.

82 ASMEQ, $A J S$, Bérichon au Devoir. 
devenir le centre de la mission puisque Szépingkai serait le lieu de résidence du préfet apostolique ${ }^{83}$. Le père Lapierre fut nommé préfet apostolique de Szépingkai, le 19 février 1930, et il prêta serment le 25 mars suivant dans la chapelle du séminaire de Moukden ${ }^{84}$. Les pères Larochelle, Bérichon et Berger firent partie du conseil de $\mathrm{M}^{\mathrm{gr}}$ Lapierre. Au point de vue financier, la préfecture reçut de l'œuvre de la Propagation de la foi une somme de cent vingt mille lires italiennes et vingt-cinq mille francs de l'œuvre de la Sainte-Enfance. La Société vota une somme de trente mille dollars pour l'administration du territoire de missions ${ }^{85}$.

Au moment de l'érection de la préfecture apostolique, le territoire où les missionnaires canadiens travaillaient depuis quatre ans comprenait douze districts, alors qu'à leur arrivée il n'y en avait que cinq. La situation des missions en 1930 témoignait du travail énorme que les prêtres canadiens avaient fourni au cours de ces quelques années d'apostolat.

Les districts de Tuchuan et de Sheng-Sin-Toun comptaient une vingtaine de chrétiens en 1926, alors qu'en 1930 il y avait six cents chrétiens et soixante catéchumènes. Ce district possédait deux églises, quatre écoles et un dispensaire. La population chrétienne du district de Hoaitensien, le plus considérable du territoire, était de huit cent vingt-cinq pratiquants. Un grand nombre d'enfants fréquentaient les écoles de la mission. Une église nouvelle avait dû être construite. A Fakousien, le missionnaire avait des difficultés avec une catégorie de chrétiens qui cherchaient à accommoder la discipline de l'Église avec leurs intérêts matériels. Le ministère n'en était pas moins fructueux. Les écoles de catéchisme et de lettres avaient toujours une assistance nombreuse et les chrétiens étaient assidus aux offices religieux. Un dispensaire devait bientôt être ouvert dans cette mission. En 1926, le poste de Léaoyuan ne comptait qu'une cinquantaine de chrétiens. Depuis, des écoles de catéchisme et de lettres avaient été construites ainsi qu'une église et un dispensaire. La population chrétienne y était nombreuse. Le père Larochelle avait ouvert, en 1928, le district de Toung-Léaosien qui avait déjà trois cent soixante-deux chrétiens. Le missionnaire avait construit une église et une école. Le premier poste ouvert par les missionnaires canadiens, Taonan, comprenait environ cent cinquante pratiquants. Cette mission avait une école, et un dispensaire devait bientôt y être installé. A Kangping, le missionnaire avait ouvert de nombreuses écoles et un dispensaire. Les chrétiens étaient nombreux, mais plusieurs étaient hostiles aux missionnaires parce que ceux-ci refusaient de se mêler des affaires civiles. Pamiencheng était un district récent qui comptait une centaine de chrétiens. Des religieuses y tenaient un dispensaire. Dans le

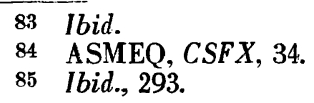


district de Szépingkai, ouvert depuis un an, il y avait une église et une école. Les chrétiens étaient environ une centaine. Le poste de Lichousien avait deux cent cinquante chrétiens et le missionnaire avait établi quelques écoles. Le nouveau poste de Tchantouvient comprenait une centaine de chrétiens ${ }^{86}$. Les missionnaires avaient prouvé au cours de ces années que la Société des Missions Étrangères de Québec était capable d'assumer seule la responsabilité de ses missions. L'érection de la préfecture confirmait ce fait. Une nouvelle étape commençait pour la Société.

\section{9 - Conclusion:}

Dix ans après sa fondation, la Société des Missions Étrangères de Québec reposait sur de solides assises. Elle était officiellement reconnue par l'État et par l'Église, et elle assurait sa propre gouverne interne selon ses constitutions qui avaient été approuvées par Rome. Au Canada, l'œuvre progressait rapidement. En 1931, le séminaire de Pont-Viau comptait trente-trois séminaristes qui se préparaient pour les missions de la Mandchourie sous la direction de cinq prêtres. Les succès remportés par les missionnaires canadiens en Mandchourie étaient aussi remarquables. Dans l'espace de cinq ans, le nombre des missionnaires s'était porté à vingt-deux. Ceux-ci avaient accompli un travail extraordinaire en réorganisant de nouveaux districts. Leurs efforts s'étaient couronnés par l'érection de leur territoire de missions en préfecture apostolique. En 1931, un petit séminaire s'établissait dans la préfecture apostolique de Szepingkai ainsi qu'un couvent pour la formation des religieuses chinoises. La préfecture fut érigée en vicariat apostolique en 1932 , avec $\mathbf{M}^{\mathrm{gr}}$ Lapierre comme vicaire apostolique ${ }^{87}$.

Jules Bernard, Missions africaines de Lyon.

s6 ASMEQ, $A J S, 1930$, Lapierre à Gauthier.

87 ASMEQ, SSME. 


\section{ABREVIATIONS}

ASMEQ Archives de la Sociëté des Missions Étrangères de la Province de Québec

CSFX Chroniques du Séminaire Saint-François-Xavier des Missions Étrangères de la Province de Québec

AME Archives de la Socièté des Missions Étrangères de la Province de Québec

AJS Articles de journaux sur la Société des Missions Étrangères de 1921 à 1933

SSME Le Séminaire et la Société des Missions Étrangères

DPVC Dossier des procès-verbaux du Comité d'organisation du Séminaire

DDRF Dossier des documents relatifs à la fondation de la Société 